\title{
Salutogenesis and Migration
}

\author{
Marguerite Daniel and Fungisai Puleng Gwanzura Ottemöller
}

\section{Introduction}

Migration involves movement across space, away from the place of origin to a new location, either in the same country (internal) or in a different country (transnational). Migration may be voluntary (as when people choose to move to seek work or for education) or forced (when violence, war or natural disasters force people to move). Migration involves numerous stressors including loss of social support networks, known environment and having to deal with uncertainty and the unfamiliar. Salutogenesis is one theory that has been used to explore how migrants cope with the stressors they experience and how they respond and adapt to new cultural contexts. In this chapter, we aim to give an overview of research on salutogenesis and migration, both forced and voluntary.

We conducted a systematic literature search using Medline, PsychINFO, CINAHL and Web of Science databases for articles published from 1999 to 2020. The thematic filters we used were: (a) Migration: migrants OR immigrants OR 'ethnic groups' OR emigration and immigration OR 'Forced migrants' OR refugee* OR 'asylum seeker*'; (b) Salutogenesis: Salutogenesis OR Salutogenic OR 'Sense of Coherence' OR SOC OR SoC OR 'Salutogenic Model of Health' OR 'Generalised resistance resources' OR GRRs OR meaningfulness OR manageability OR comprehensibility; and (c) Population: Child OR Children OR Women OR Woman OR Men OR Man OR Family OR Families OR Adolescent* OR 'Young adults' OR Adults. The results of these searches, however, excluded relevant articles and book chapters of which we were aware, so we also used Google Scholar to extend the search, using the same filters and period. In addition to some peer-reviewed publications not found by the other databases which we have included in our analysis, Google Scholar also disclosed Master's and PhD theses that we have not included.

M. Daniel $(\varangle)$ · F. P. G. Ottemöller

Department of Health Promotion and Development, Faculty of

Psychology, University of Bergen, Bergen, Norway

e-mail: Marguerite.Daniel@uib.no; Fungi.Ottemoller@uib.no
Table 47.1 Criteria used for analysing content of articles found in literature search

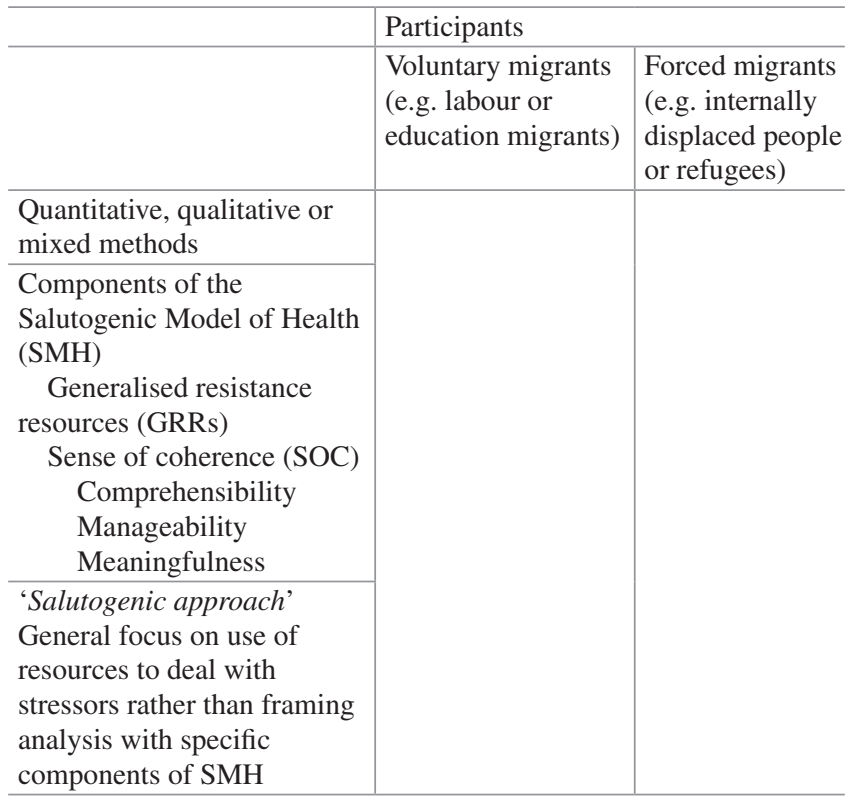

We categorised the articles identified by the searches using the criteria shown in Table 47.1.

Most articles on salutogenesis and migration use welldefined components of the Salutogenic Model of Health (SMH) such as generalised resistance resources (GRRs) and sense of coherence (SOC). However, several articles use a 'salutogenic approach' by which we mean a general focus on the use of resources to deal with stressors without specifically using components of SMH. There were a few articles that did not neatly fit into the categories, but did in some way deal with salutogenesis and cultural diversity. In the end, we included 59 peer-reviewed articles in our analysis. The vast majority of articles are quantitative and use the SOC-29 or the SOC-13 scale, for example, as part of a group of statistical measures. We excluded articles that measured the SOC of children (who had not migrated) and whose parents were migrants. We identified 33 articles on forced migration, 17 on voluntary migration, 6 where both forced and voluntary migrants were included and 3 articles where it was not 
specified (for example, participants described as 'migrants at risk of social exclusion').

Before moving on to the overview of articles on salutogenesis and voluntary and forced migration, we present a couple of articles that cannot be classified as either, but that we consider relevant for the topic of salutogenesis and migration. They are more theoretical than empirical but provide valuable insights into the cultural context of migrants. Benz et al. (2014) analyse all texts in English by Antonovsky that have any reference to 'culture'. They identified cultural stressors such as minority background, rapid change in culture, cultural discrimination and hostile or complex culture. They also identified cultural assets or GRRs such as cultural stability, being valued in a culture, integration, cultural adaptability and 'culture gives place in the world' (Benz et al., 2014). These are all important in shaping the SOC of refugees, displaced people, labour migrants or international students. A paper by Riedel et al. (2011), in the context of the rising numbers of migrants, develops a theoretical framework that integrates salutogenesis and an acculturation model by Berry (2006). Acculturation stress refers to challenges arising from contact between cultures that cannot easily be resolved. In their theoretical framework, the authors show how the GRRs and SOC of migrants helps them cope with acculturation stress (Riedel et al., 2011).

\section{Salutogenesis and Voluntary Migration}

Voluntary migration focuses on people who have willingly left their home countries, city or region of origin to either work or study. This section presents empirical articles that focus on internal and external voluntary migration.

\section{Internal Voluntary Migration}

Internal voluntary migration occurs when people migrate within their home country in order to either work or study. We found only two articles on internal voluntary migration, both related to labour migration. The first is a quantitative study from China. Chen et al. (2019) look at internal migrant workers in China to identify the potential mechanisms of psychological well-being. The SOC-13 scale is one of the instruments used to assess the migrants' depression, subjective well-being and SOC. The authors find that in general migrant workers have poor mental health and weak SOC, but they conclude that GRRs (income ratio, marital status) are positively associated with higher SOC and may strengthen SOC in this group (Chen et al., 2019). The second article about internal voluntary migration is a qualitative study from Ghana that focuses on women who had migrated from northern regions in Ghana to work as porters in the capital city,
Accra (Wilson \& Mittelmark, 2013). The study adopts a 'salutogenic perspective' and the authors note that some of the migrants adjust well in spite of difficult circumstances. GRRs include religious beliefs, achieving financial stability and developing and cultivating supportive social networks (Wilson \& Mittelmark, 2013).

\section{External Labour Migration}

External labour migration involves people leaving their home country to find work in a different country. The majority of articles we found on voluntary migration focus on external labour migration and most were quantitative.

There is a cluster of three quantitative articles on migrants from the former Soviet Union (FSU) living in Israel. Soskolne's (2001) study examines whether the combination of single parenthood and occupational drift in the context of migration implies greater risk of psychological distress for single mothers compared to married mothers from FSU. Soskolne concludes that single mothers had weaker SOC and higher levels of distress than married mothers. The beneficial effects of social support in reducing distress were significantly greater for married than for single immigrant mothers from the FSU living in Israel (Soskolne, 2001). Sagy et al. (2009) examine SOC as a protective factor towards drug and alcohol abuse amongst Israel-born and immigrant teenagers from the FSU. They use both the SOC13 scale and the Sense of family coherence scale. Although both groups had low levels of interest in using drugs or alcohol to cope with stress, the study found that the immigrant group had a more permissive attitude. Further, they found scoring strongly on personal and family SOC to be protective against drug and alcohol abuse (Sagy et al., 2009). Ponizovsky-Bergelson et al.' (2015) study focuses on filial responsibility amongst young immigrants from FSU living in Israel. Filial responsibility due to migration occurs when young members of a migrant family take responsibility and try to help their parents to cope with difficulties related to migration. They used SOC-29 as one of a series of measures of resilience (others included optimism and social support) and found that SOC is directly protective of psychological adjustment in the context of filial responsibility (PonizovskyBergelson et al., 2015).

Two studies from the same team of authors in Germany conduct comparative studies about migrants from Turkey and Poland and the native populations in these countries. Erim et al. (2011) compare two clinical samples of outpatients with neurotic disorders: one sample of Turkish immigrant outpatients living in Germany and one sample of Turkish outpatients in Istanbul. They applied the SOC-29 questionnaire to both groups. Both samples had weaker SOC scores compared to the Turkish population, the German 
population and the German population with similar neurotic disorders, but there were no differences between the two samples (Erim et al., 2011). The authors found SOC to be a strong predictor of depressiveness. They attributed this to the collectivist nature of the Turkish people and their tendency to attribute the skills measured by the SOC scale to their families, and not themselves as individuals, which resulted in weak scores (Erim et al., 2011). Morawa and Erim (2015) compare the health-related quality of life and sense of coherence amongst Polish immigrants in Germany, Poles in Poland and the German population. The authors report that SOC levels are weaker in both Polish samples compared to the German population. They explain that this could be due to cultural differences, as the individualistic attributes measured in the SOC questionnaire do not correspond with the collectivistic orientation amongst Polish people (Morawa \& Erim, 2015).

There are two papers based on a study on Pakistani migrants living in Toronto, Canada. Jibeen and Khalid (2010) examine how coping resources (SOC and perceived social support) and coping strategies (both problem- and emotionfocused) have an impact on positive well-being. They found that, along with a higher level of perceived income comfort, coping resources and strategies were associated with higher positive functioning. Jibeen (2011) found that sense of coherence and perceived social support moderated the effect of acculturative stress and enabled positive functioning in terms of self-acceptance and positive relations with others.

A quantitative study on migrants in Sweden includes both forced and voluntary migrants. Sundquist et al. (2000) examine the impact of several variables including exposure to violence, SOC, acculturation, sense of control over one's life, economic difficulties and education on psychological distress in immigrants. Their sample includes immigrants from Iran, Chile, Poland, Turkey and those of Kurdish descent. The study uses data from the first Swedish national survey of immigrants. The study uses only three questions from the SOC-13 item questionnaire, one each to measure comprehensibility, manageability and meaningfulness. The authors found that a weak SOC was related to migration status and psychological distress (Sundquist et al., 2000).

We found only a few mixed methods or qualitative studies on salutogenesis and labour migration. In a mixed-methods study, Slootjes et al. (2017) examine the development of a strong sense of coherence and the role of migration, integration and GRRs in this process. A sample of women with Turkish, Moroccan and Surinamese backgrounds completed the SOC-13-item questionnaire. The women also participated in interviews where they were asked to share life stories and reflect on their migration and integration experiences in the Netherlands. Slootjes et al. (2017) conclude that consistency and load balance were associated with a strong SOC amongst these women. However, the women did not neces- sarily require decision-making power to develop meaningfulness. The findings indicate that migration and integration are related to the mechanisms shaping SOC, but that this depends on the availability and use of GRRs (Slootjes et al., 2017). Slootjes et al. (2018) report on a second study with the same sample, but this time including Dutch women. They use SOC to examine how the women escape the vicious cycle between health problems and unemployment (Slootjes et al., 2018, p. 1). The study moves away from the focus of how SOC influences health, to examine how SOC functions and whether it is applicable outside the health domain (Slootjes et al., 2018, p. 11). The findings indicate that the participants found it possible to escape the vicious cycle between health problems and unemployment by focusing on meaning and the purpose of adversity. Slootjes et al. (2018) found that stronger levels of SOC were associated with narratives of meaningful endurance.

We identified three qualitative studies using salutogenesis to explore different aspects of labour migration. Crowther and Lau (2019) explore how communication influences maternity care amongst migrant Polish women in Scotland. They interviewed nine women who had recently used the maternity services, and then adopted a salutogenic conceptual framework to analyse the data. Their findings identify the significance of quality communication, relationship and culturally sensitive practices as ways of mitigating feelings of vulnerability amongst Polish women in Scotland. SOC facilitates 'the women's ability to comprehend and their capacity to understand their own experiences of communication challenges' (Crowther \& Lau, 2019, p. 30). Using a salutogenic approach, Obrist and Büchi (2008) investigate why West and East African migrants in Switzerland stay healthy. The meanings of health and resilience are explored using case studies and focus group discussions. Although the study claims to use a salutogenic approach, salutogenesis is not really mentioned much in the article. The focus of the research is more on resilience, and stress as an idiom for resilience that helps the migrants to reflect, interpret and explain their feelings (Obrist \& Büchi, 2008). Bürgelt et al. (2008) investigate how German couples, who migrated to New Zealand, experience and interpret the migration process. They interviewed four couples who chose to settle permanently in New Zealand and four couples who returned to Germany. What the authors call 'the salutogenic paradigm' highlights how successful adaptation to migration depends on the meaning migrants find in their experiences, which leads to well-being and growth (Bürgelt et al., 2008).

\section{External Migration for Education}

We found only three papers where participants had migrated internationally for purposes of education. Interestingly, all 
the studies had a comparative aspect. Ying et al. (2000, 2007) conducted two studies with American-born and immigrant Chinese students. In their first study, they investigated whether cultural orientation and racial discrimination influenced subjective competence (as measured by sense of coherence: SOC-29) in these two groups (Ying et al., 2000). Their results indicate that Chinese American students tend to be more integrated into American society and have a stronger SOC than their immigrant counterparts who are more bi-cultural. Although, racial discrimination negatively impacted both groups' SOC, the immigrant group were better able to cope and distance themselves from it. This was attributed to their not being as well-integrated into the American way of life and, therefore, not having grown up with the expectation of attaining the American dream (Ying et al., 2000). The second study examined SOC as a mediator between depressive symptoms, parent and peer attachment and college challenges, to see whether there was variation depending on migration status (Ying et al., 2007). The sample included Chinese American students and compared them with two immigrant groups: early immigrants and late immigrants, i.e. immigrants who had been in the United States longer and those who had arrived more recently. The findings indicated that peer and parental attachment had different effects on the various groups' depressive symptoms and SOC; moreover, SOC mediated the effect of attachment on depressive symptoms in all three groups (Ying et al., 2007). Grayson (2008) used the SOC-13-item questionnaire in his study to examine the impact of SOC on academic achievement between domestic and international students in Canadian universities. The study found that international students with a strong SOC were more likely to perform well academically than those with a weak SOC (Grayson, 2008).

In summary, the papers reviewed above indicate that quantitative studies dominate research with voluntary migrants, and the predominant focus is pathogenic - mental health with an emphasis on depression. Moreover, some of the studies indicate that the SOC scale is not always sufficient to capture the experiences of populations with more collectivist attributes, and they are portrayed as having weak SOC (Erim et al., 2011; Morawa \& Erim, 2015). The qualitative studies we identified, although few, had a broader focus on life experiences and a more salutogenic emphasis.

\section{Salutogenesis and Forced Migration}

Forced migration occurs when people flee war, persecution, violence or natural disasters. Sometimes they move to a different part of their own country and are then known as internally displaced persons (IDPs). When they flee beyond the borders of their country, they are refugees.

\section{Salutogenesis and Internally Displaced Persons}

All the articles we found on salutogenesis and IDPs report quantitative research. The studies cover six countries: three in Sub-Saharan Africa, namely Eritrea, Niger and Democratic Republic of the Congo (DRC), and then Palestine and Chechnya, and finally, two studies in Indonesia by the same team of authors. Aitcheson et al. (2017) used the 26-item family sense of coherence scale (i.e. the extent to which a family collectively perceives the world as comprehensible, manageable and meaningful) as one of four family-level predictors of health and well-being in Palestinian adolescents living in Gaza (although their outcome measures were depression and anxiety). They found that a strong family SOC, along with several other family, cultural and individual factors, contributed to adolescent health. Almedom et al. (2005a, b, 2007) produced a cluster of articles based on a study with Eritrean IDPs. They used only SOC-13 and found that IDPs had a significantly weaker SOC than persons who had not been displaced and that women were significantly more negatively affected than men. Their method of data collection included some 'qualitative' discussion on some of the 13 items. In a publication on IDPs in Chechnya by Parker et al. (2013), in which Almedom is also a co-author, they called the methodology of the Eritrean study 'Sense and Sensibilities of Coherence (SSOC) Methodology'. They found that the meaningfulness scores were significantly higher than comprehensibility and manageability scores, with hope emerging as the key component of meaningfulness. In a study in the DRC by Pham et al. (2010), the authors set out to investigate the association of SOC with prolonged and cumulative exposure to traumatic events and violence. Of the total of 2635 respondents, $2139(85.5 \%)$ were displaced, and they had a significantly weaker SOC than those who were not displaced. Likewise, exposure to a cumulative number of traumatic or violent incidents was inversely related to SOC. The authors suggest that, given that SOC is a protective factor, finding ways to strengthen it by providing an environment that is comprehensible, manageable and meaningful could enhance health and well-being of internally displaced persons. They suggest that interventions, particularly in IDP camps, that help people living there gain a sense of control over security, shelter, food and clothing, would be a good place to start. However, they acknowledge that the best solution would be peace and stability. The Indonesian studies follow the same group of IDPs over time using SOC-13 as an indicator of mental health. In the first study (Turnip et al., 2010), the IDPs suffered significantly more psychological distress than a control group of nonIDPs, and the authors concluded that displacement is a significant risk factor for distress. The second study (Turnip et al., 2016) reported data collected a year after the first study 
and found that for IDPs, despite previous traumatic experiences, both SOC and well-being had improved. The authors attribute this to improved socio-economic conditions. Veronese et al. (2019) worked in a 'salutogenic tradition', or what we have called a 'salutogenic approach', focussing on the social ecology of adolescents in IDP camps in Niger. Despite multiple traumatic experiences, the adolescents functioned positively by using relational resources and selfcompetence. We found no qualitative studies on salutogenesis and IDPs.

\section{Salutogenesis and Refugees}

There seems to be a growing interest in using SMH or the salutogenic approach in studying the health and well-being of refugees. Although we have included articles for the first two decades of the twenty-first century, more than half of the articles in this section were written since 2015, the start of the so-called refugee 'crisis'. The literature on salutogenesis and refugees is dominated by studies from Sweden (which, with one exception, are quantitative), both before and after the refugee crisis. In the Swedish studies, before 2015, the refugees were from Iran (Bayard-Burfield et al., 2001; Ghazinour et al., 2004; Lindencrona et al., 2008) and the Balkans (Björn et al., 2013; Roth \& Ekblad, 2006). After 2015, Syrians and Afghans (Sarkadi et al., 2018) are the dominant refugee populations with some from Eritrea (Bergstrom-Wuolo et al., 2018). The remaining studies are conducted mainly in Global North countries (Norway, Finland, Denmark, Germany, Canada, Australia, United States and Israel). They are mostly qualitative in approach, except for three recent articles from Germany (Georgiadou et al., 2020; Kindermann et al. 2020a, b) and a series of three articles by Getnet and colleagues (Getnet \& Alem, 2019a, b; Getnet et al., 2019) conducted with Eritrean refugees in Ethiopia.

The studies using SMH or its components are mostly quantitative in methodology. Only the study by Ghazinour et al. (2004) uses the SOC-29 scale; the rest use the SOC-13 scale or some adaptation of this shorter scale. It is mainly the older studies that use adaptations, for example, BayardBurfield et al. (2001) and Lindencrona et al. (2008) use only 3 of the SOC-13 items while Roth \& Ekblad, (2006) use 12 items. Two recent studies from Germany (Kindermann et al. 2020a, b) use SOC-9L (Leipzig short scale). Getnet \& Alem, (2019a) use SOC-13 but found that the removal of item 2, 'Has it happened in the past that you were surprised by the behaviour of people whom you thought you knew well?', improved the measurement of SOC in Eritreans.

A surprising number of the quantitative studies, although using SOC as one of a number of measures, are essentially measuring depression, anxiety or PTSD - i.e. pathological measures - rather than health and well-being. Again, this applies mostly to the older studies, for example, the BayardBurfield et al. (2001) study focused on psychiatric illness, the Lindencrona et al. (2008) study focused on mental disorder and stress and the Roth \& Ekblad, (2006) study found that for Kosovan refugees over time, depression increased while SOC decreased. Kuittinen et al. (2014) examined the manifestation of depression and psychosocial correlates amongst older Somali refugees and native Finns. Likewise, two of the Ethiopian studies (Getnet \& Alem, 2019b; Getnet et al., 2019) focused on pathological outcomes like depression and PTSD. The Ghazinour et al. (2004) article, although old, focused on health and well-being outcomes like social support and coping. The authors found that meaningfulness was particularly important in helping refugees cope with trauma and have a good quality of life.

Most of the more recent quantitative studies on salutogenesis and refugees focus on health and well-being. Georgiadou et al. (2020) explored the role of SOC along with other measures of quality of life for Syrian refugees in Germany separated from their marital partners, and Kindermann et al. (2020b) examined the link between SOC and utilisation of health care. Some studies measure SOC after the study population has experienced a health promotion intervention. Arola et al. (2018) worked with older refugees from the Balkans while Sarkadi et al. (2018) studied unaccompanied minors from Afghanistan and Syria; both studies found a stronger SOC which helped participants cope with stress and trauma. A recent study with Syrian adolescents in refugee camps in Israel (Braun-Lewensohn \& Al-Sayed, 2018) found that gender (being a girl) and length of time in the camp contributed most to stress and psychological problems. Still, their role almost disappeared once SOC was taken into account. In other words, SOC was found to be the most important factor in reducing stress and psychological problems. A study with women in refugee camps in Greece, which used SOC-13 and a 16-item measure of community SOC, found that SOC mediates negative variables (e.g. time spent in camps) and outcomes such as reduced depression and anxiety (Braun-Lewensohn et al., 2019).

We found ten qualitative studies on salutogenesis and refugees, four using components of SMH and six using a salutogenic approach. While many of the qualitative studies focused on the health and well-being of the participants, some studies looked at other aspects of participants' lives. The de Wal Pastoor (2015, p. 247) study in Norway views schools as 'salutogenic arenas' for young refugees spaces that can provide safety and predictability, resources to cope and can promote meaning. The study findings show that the support received tends to be somewhat random. Still, the author concluded that schools are poten- 
tially supportive environments for young refugees - in contrast to therapeutic interventions. In another study with a school setting, this time in Canada, Reimer (2020) explores how restorative justice helps students to build a strong sense of coherence, both individual and collective. She defines restorative justice as 'a set of principles and practices that positions harm as a violation of people and relationships rather than a violation of rules and laws' (Reimer, 2020, p. 410). Another article adopting an unusual approach is the one by Kolanen et al. (2016), which explores how components of SOC can improve breastfeeding amongst Somali refugees in Finland. Given that new mothers in the Somali community often cease breastfeeding prematurely, the authors explored new mothers' GRRs, comprehensibility, manageability and meaningfulness. They found the new mothers were motivated to breastfeed by religious and cultural factors, with their most important source of information being their own mother and other female relatives. The authors conclude that the GRRs that emerged in the study could be exploited during health service counselling to increase effectiveness.

Qualitative studies are also able to explore the role of families and communities. Two studies in Sweden examined the SOC of parents. Atwell et al. (2009) found that while parents experienced numerous stressors and felt they were not coping, they had a strong sense of purpose (meaningfulness) for their children. Björn et al. (2013) took a salutogenic approach in running family therapy sessions and found that cohesive family narratives about traumatic experiences and their new lives emerged. A recent study in Denmark (Poulsen et al., 2020) uses the salutogenic approach to explore how the natural environment and participation in an eco-village can help severely traumatised refugees. Horticultural activities and respectful attitude of staff contributed to recovery while new skills were achieved, and feelings of isolation decreased (Poulsen et al., 2020, p. 1). The salutogenic approach has also been used in life course studies amongst refugees in a study on perceptions of health amongst older refugees from the Balkans living in Sweden (Lood et al., 2016).

One GRR that emerges in several qualitative studies is spirituality or religious beliefs. Sossou et al. (2008) worked with Bosnian refugees in the United States. They conducted narrative interviews after a quantitative study - and purposely selected women with a strong SOC. Spirituality was amongst the GRRs they identified, along with social support and services. Two publications by the same team of authors in Australia working with Burmese refugees found spiritual and religious beliefs as well as individual and cultural strengths and values, and duty to family and community, all contributed to the ability to cope (Borwick et al., 2013; Shakespeare-Finch et al., 2014).

\section{Health Promotion Interventions for Migrants}

Salutogenesis, particularly through the identification of GRRs, provides an approach for designing health promotion interventions. We found two pairs of studies that report on formative research for a health promotion intervention with migrant women, and then evaluate the intervention. In Norway, an intervention for new mothers in a multi-ethnic community was planned and evaluated using salutogenic theory (Leirbakk et al., 2018, 2019). In Spain, BonmatiTomas et al. (2016) report on a qualitative study mapping the assets of immigrant women experiencing health inequities and at risk of social exclusion. They use this knowledge to develop better tools for a health promotion programme. In a second article (Bonmatí-Tomas et al., 2019), they report on a quantitative evaluation of the health promotion intervention. Although the SOC-13 scores did not change significantly after the intervention, the participants did experience less perceived stress (Bonmatí-Tomas et al., 2019).

\section{How Does Salutogenesis Contribute to Research with Refugees?}

Many of the papers included in this study state that they are using SOC as a measure of coping or of resilience. So does the use of salutogenesis in migration studies add anything that other strengths-based theories could not?

To answer this question, we need to return to Antonovsky's (1996) paper that suggests the use of SMH as a theory for health promotion. He contends that the prevailing axiom of a dichotomous classification of people as either healthy or temporarily, permanently or fatally ill (in contrast to his view that all humans are somewhere along a healthy/dis-ease continuum) leads to a concern with pathogens and risk factors. Antonovsky (1996) points out that it is not only curative medicine and preventative medicine that take this stance, but even health promoters (Antonovsky sees this as a failure of nerve!). Unfortunately, we have found ample evidence of this pathogenic approach in the literature on salutogenesis and migration. It is mainly the older, quantitative papers that focus on pathogenic outcomes such as depression or anxiety or PTSD - despite including some measure of SOC. Antonovsky (1996) warned that a focus on pathology is often narrow and does not in any way take the rich complexity of human life into account.

The SOC, comprised simultaneously of cognitive, behavioural and motivational elements, can deal with complexity. Some of the more recent literature on salutogenesis and migration has succeeded in taking a salutogenic approach, with a focus on what Antonovsky (1996) calls 'salutary' 
factors. This has enabled innovative studies on the health and well-being of migrants from a more holistic perspective. One example is the identification of cultural values and practices that may be used as GRRs (Borwick et al., 2013; Kolanen et al., 2016; Sossou et al., 2008). Creating 'salutogenic arenas' in schools to help unaccompanied minors move towards greater health (de Wal Pastoor, 2015) and training children in restorative justice (Reimer, 2020) are examples in school settings. Understanding how flexibility in expectations can enhance SOC (Slootjes et al., 2017) provides a pathway for interventions that strengthen SOC. Another innovative approach to the holistic health and well-being of refugees is training in horticultural skills within eco-villages (Poulsen et al., 2020). It is often qualitative studies that allow innovative use of salutogenesis in the study of refugees. Antonovsky (1996, p. 15) promotes SMH as a systematic theoretical guide for research and action. We have included a few papers that use salutogenesis to plan and evaluate interventions (Bonmati-Tomas et al., 2016, 2019; Leirbakk et al., 2018, 2019).

In conclusion, while some of the earlier research tended to focus on pathogenic outcomes and, therefore, does not contribute much to studying migration from a salutogenic perspective, more recent papers focus on salutary factors, take a more holistic view and illustrate innovative ways of using salutogenesis to explore migration experiences. This indicates an encouraging move towards migration research that is more in line with Antonovsky's interest in what builds health.

\section{References}

Aitcheson, R. J., Abu-Bader, S. H., Howell, M. K., Khalil, D., \& Elbedour, S. (2017). Resilience in Palestinian adolescents living in Gaza. Psycholological Trauma, 9(1), 36-43. https://doi. org/10.1037/tra0000153

Almedom, A. M., Tesfamichael, B., Mohammed, Z., Mascie-Taylor, N., Muller, J., \& Alemu, Z. (2005a). Prolonged displacement may compromise resilience in Eritrean mothers. African Health Sciences, 5(4), 310-314. https://doi.org/10.5555/afhs.2005.5.4.310

Almedom, A. M., Tesfamichael, B., Mohammed, Z., Muller, J., MascieTaylor, N., \& Alemu, Z. (2005b). "Hope" makes sense in Eritrean sense of coherence, but "loser" does not. Journal of Loss and Trauma, 10(5), 433-451.

Almedom, A. M., Tesfamichael, B., Saeed Mohammed, Z., MascieTaylor, C. G., \& Alemu, Z. (2007). Use of 'sense of coherence (SOC)' scale to measure resilience in Eritrea: Interrogating both the data and the scale. Journal of Biosocial Science, 39(1), 91-107. https://doi.org/10.1017/s0021932005001112

Antonovsky, A. (1996). The salutogenic model as a theory to guide health promotion. Health Promotion International, 11(1), 11-18.

Arola, L. A., Barenfeld, E., Dahlin-Ivanoff, S., \& Haggblom-Kronlof, G. (2018). Distribution and evaluation of sense of coherence among older immigrants before and after a health promotion intervention - Results from the RCT study promoting aging migrants' capability. Clinical Interventions in Aging, 13, 2317-2328. https://doi. org/10.2147/cia.s177791
Atwell, R., Gifford, S. M., \& McDonald-Wilmsen, B. (2009). Resettled refugee families and their children's futures: Coherence, hope and support. Journal of Comparative Family Studies, 40(5), 677-697.

Bayard-Burfield, L., Sundquist, J., \& Johansson, S. E. (2001). Ethnicity, self reported psychiatric illness, and intake of psychotropic drugs in five ethnic groups in Sweden. Journal of Epidemiology and Community Health, 55(9), 657-664. https://doi.org/10.1136/ jech.55.9.657

Benz, C., Bull, T., Mittelmark, M., \& Vaandrager, L. (2014). Culture in salutogenesis: The scholarship of Aaron Antonovsky. Global Health Promotion, 21(4), 16-23.

Bergstrom-Wuolo, M., Dahlström, J., Hertting, K., \& Kostenius, C. (2018). My heart has no hurt: The health of young immigrants. International Journal of Migration, Health and Social Care, 14(3), 290-304.

Berry, J. W. (2006). Stress perspectives on acculturation. In D. L. Sam \& J. W. Berry (Eds.), The Cambridge handbook of acculturation psychology (pp. 43-57). Cambridge University Press.

Björn, G. J., Gustafsson, P. A., Sydsjö, G., \& Bertero, C. (2013). Family therapy sessions with refugee families; a qualitative study. Conflict and Health, 7(1), 7. https://doi.org/10.1186/1752-1505-7-7

Bonmatí-Tomas, A., Malagón-Aguilera, M., Gelabert-Vilella, S., Bosch-Farré, C., Vaandrager, L., García-Gil, M., \& Juvinyà-Canal, D. (2019). Salutogenic health promotion program for migrant women at risk of social exclusion. International Journal for Equity in Health, 18(1), 1-9.

Bonmati-Tomas, A., Malagon-Aguilera Mdel, C., Bosch-Farre, C., Gelabert-Vilella, S., Juvinya-Canal, D., \& Garcia Gil Mdel, M. (2016). Reducing health inequities affecting immigrant women: A qualitative study of their available assets. Globalization and Health, 12(1), 37. https://doi.org/10.1186/s12992-016-0174-8

Borwick, S., Schweitzer, R. D., Brough, M., Vromans, L., \& Shakespeare-Finch, J. (2013). Well-being of refugees from Burma: A salutogenic perspective. International Migration, 51(5), 91-105.

Braun-Lewensohn, O., Abu-Kaf, S., \& Al-Said, K. (2019). Women in refugee camps: Which coping resources help them to adapt? International Journal of Environmental Research and Public Health, 16(3990), 1-11. https://doi.org/10.3390/ijerph16203990

Braun-Lewensohn, O., \& Al-Sayed, K. (2018). Syrian adolescent refugees: How do they cope during their stay in refugee camps? Frontiers in Psychology, 9, 1258. https://doi.org/10.3389/fpsyg.2018.01258

Bürgelt, P. T., Morgan, M., \& Pernice, R. (2008). Staying or returning: Pre-migration influences on the migration process of German migrants to New Zealand. Journal of Community \& Applied Social Psychology, 18(4), 282-298.

Chen, H., Wang, L., Wei, Y., Ye, B., Dai, J., Gao, J., ... Fu, H. (2019). The potential psychological mechanism of subjective wellbeing in migrant workers: A structural equation models analysis. International Journal of Environmental Research and Public Health, 16(12), 2229.

Crowther, S., \& Lau, A. (2019). Migrant Polish women overcoming communication challenges in Scottish maternity services: A qualitative descriptive study. Midwifery, 72, 30-38. https://doi. org/10.1016/j.midw.2019.02.004

de Wal Pastoor, L. (2015). The mediational role of schools in supporting psychosocial transitions among unaccompanied young refugees upon resettlement in Norway. International Journal of Educational Development, 41, 245-254.

Erim, Y., Morawa, E., Atay, H., Aygun, S., Gokalp, P., \& Senf, W. (2011). Sense of coherence and depression in the framework of immigration: Turkish patients in Germany and in Turkey. International Review of Psychiatry, 23(6), 542-549. https://doi. org/10.3109/09540261.2011.637908

Georgiadou, E., Schmitt, G. M., \& Erim, Y. (2020). Does the separation from marital partners of Syrian refugees with a residence permit in Germany have an impact on their quality of life? Journal 
of Psychosomatic Research, 130, 1-7. https://doi.org/10.1016/j. jpsychores.2020.109936

Getnet, B., \& Alem, A. (2019a). Construct validity and factor structure of sense of coherence (SoC-13) scale as a measure of resilience in Eritrean refugees living in Ethiopia. Conflict and Health, 13, 3. https://doi.org/10.1186/s13031-019-0185-1

Getnet, B., \& Alem, A. (2019b). Validity of the Center for Epidemiologic Studies Depression Scale (CES-D) in Eritrean refugees living in Ethiopia. BMJ Open, 9(5), e026129. https://doi.org/10.1136/ bmjopen-2018-026129

Getnet, B., Medhin, G., \& Alem, A. (2019). Symptoms of posttraumatic stress disorder and depression among Eritrean refugees in Ethiopia: Identifying direct, meditating and moderating predictors from path analysis. BMJ Open, 9(1), e021142. https://doi. org/10.1136/bmjopen-2017-021142

Ghazinour, M., Richter, J., \& Eisemann, M. (2004). Quality of life among Iranian refugees resettled in Sweden. Journal of Immigrant Health, 6(2), 71-81.

Grayson, J. P. (2008). Sense of coherence and academic achievement of domestic and international students: A comparative analysis. Higher Education, 56(4), 473-492.

Jibeen, T. (2011). Moderators of acculturative stress in Pakistani immigrants: The role of personal and social resources. International Journal of Intercultural Relations, 35(5), 523-533.

Jibeen, T., \& Khalid, R. (2010). Predictors of psychological well-being of Pakistani immigrants in Toronto, Canada. International Journal of Intercultural Relations, 34(5), 452-464.

Kindermann, D., Zeyher, V., Nagy, E., Brandenburg-Ceynowa, H., Junne, F., Friederich, H.-C., ... Nikendei, C. (2020a). Predictors of asylum seekers' mental health course in the early stages of resettlement: Results of a longitudinal study. Journal of Psychosomatic Research 132, 1-10. https://doi.org/10.1016/j.jpsychores.2020.109977

Kindermann, D., Zeyher, V., Nagy, E., Friederich, H.-C., Bozorgmehr, K., \& Nikendei, C. (2020b). Predictors of asylum seekers' health care utilization in the early phase of resettlement. Frontiers in Psychiatry, 11(475), 1-12. https://doi.org/10.3389/fpsyt.2020.00475

Kolanen, H., Välimäki, T., Vehviläinen-Julkunen, K., Hemberg, J., Eriksson, K., Nyström, L., ... Kafkia, T. (2016). Breastfeeding among Somali mother's living in Finland under salutogenic approach. International Journal of Caring Sciences, 9(2), 384.

Kuittinen, S., Punamäki, R.-L., Mölsä, M., Saarni, S. I., Tiilikainen, M., \& Honkasalo, M.-L. (2014). Depressive symptoms and their psychosocial correlates among older Somali refugees and native Finns. Journal of Cross-Cultural Psychology, 45(9), 1434-1452.

Leirbakk, M. J., Magnus, J. H., Torper, J., \& Zeanah, P. (2019). Look to Norway: Serving new families and infants in a multiethnic population. Infant Mental Health Journal, 40(5), 659-672. https://doi. org/10.1002/imhj.21804

Leirbakk, M. J., Torper, J., Engebretsen, E., Opsahl, J. N., Zeanah, P., \& Magnus, J. H. (2018). Formative research in the development of a salutogenic early intervention home visiting program integrated in public child health service in a multiethnic population in Norway. BMC Health Services Research, 18(1), 741.

Lindencrona, F., Ekblad, S., \& Hauff, E. (2008). Mental health of recently resettled refugees from the Middle East in Sweden: The impact of pre-resettlement trauma, resettlement stress and capacity to handle stress. Social Psychiatry and Psychiatric Epidemiology, 43(2), 121-131.

Lood, Q., Häggblom-Kronlöf, G., \& Dellenborg, L. (2016). Embraced by the past, hopeful for the future: Meaning of health to ageing persons who have migrated from the Western Balkan region to Sweden. Ageing \& Society, 36(3), 649-665.

Morawa, E., \& Erim, Y. (2015). Health-related quality of life and sense of coherence among Polish immigrants in Germany and indigenous Poles. Transcultural Psychiatry, 52(3), 376-395. https://doi. org/10.1177/1363461514565851
Obrist, B., \& Büchi, S. (2008). Stress as an idiom for resilience: Health and migration among sub-Saharan Africans in Switzerland. Anthropology \& Medicine, 15(3), 251-261.

Parker, J., Tiberi, L. J., Akhilgova, J., Toirov, F., \& Almedom, A. M. (2013). "Hope is the engine of life"; "Hope dies with the person": Analysis of meaning making in FAO-supported North Caucasus communities using the "Sense and Sensibilities of Coherence" (SSOC) methodology. Journal of Loss and Trauma, 18(2), 140-151.

Pham, P. N., Vinck, P., Kinkodi, D. K., \& Weinstein, H. M. (2010). Sense of coherence and its association with exposure to traumatic events, posttraumatic stress disorder, and depression in eastern Democratic Republic of Congo. Journal of Traumatic Stress, 23(3), 313-321.

Ponizovsky-Bergelson, Y., Kurman, J., \& Roer-Strier, D. (2015). Adjustment enhancer or moderator? The role of resilience in postmigration filial responsibility. Journal of Family Psychology, 29(3), 438.

Poulsen, D. V., Pálsdóttir, A. M., Christensen, S. I., Wilson, L., \& Uldall, S. W. (2020). Therapeutic nature activities: A step toward the labor market for traumatized refugees. International Journal of Environmental Research and Public Health, 17(20), 1-19. https:// doi.org/10.3390/ijerph17207542

Reimer, K. E. (2020). "Here, it's like you don't have to leave the classroom to solve a problem": How restorative justice in schools contributes to students' individual and collective sense of coherence. Social Justice Research, 33, 406-427. https://doi.org/10.1007/ s11211-020-00358-5

Riedel, J., Wiesmann, U., \& Hannich, H.-J. (2011). An integrative theoretical framework of acculturation and salutogenesis. International Review of Psychiatry, 23(6), 555-564. https://doi.org/10.3109/0954 0261.2011 .637912

Roth, G., \& Ekblad, S. (2006). A longitudinal perspective on depression and sense of coherence in a sample of mass-evacuated adults from Kosovo. Journal of Nervous \& Mental Disease, 194(5), 378-381. https://doi.org/10.1097/01.nmd.0000217882.70120.38

Sagy, S., Shani, E., \& Leibovich, E. (2009). Factors related to attitudes towards drug use and alcohol drinking: Comparing immigrants from the former Soviet Union and Israeli-born adolescents. Journal of Substance Use, 14(1), 10-18.

Sarkadi, A., Ådahl, K., Stenvall, E., Ssegonja, R., Batti, H., Gavra, P., ... Salari, R. (2018). Teaching recovery techniques: Evaluation of a group intervention for unaccompanied refugee minors with symptoms of PTSD in Sweden. European Child \& Adolescent Psychiatry, 27(4), 467-479.

Shakespeare-Finch, J., Schweitzer, R. D., King, J., \& Brough, M. (2014). Distress, coping, and posttraumatic growth in refugees from Burma. Journal of Immigrant \& Refugee Studies, 12(3), 311-330.

Slootjes, J., Keuzenkamp, S., \& Saharso, S. (2017). The mechanisms behind the formation of a strong Sense of Coherence (SOC): The role of migration and integration. Scandinavian Journal of Psychology, 58(6), 571-580. https://doi.org/10.1111/sjop.12400

Slootjes, J., Keuzenkamp, S., \& Saharso, S. (2018). Narratives of meaningful endurance - How migrant women escape the vicious cycle between health problems and unemployment. Comparative Migration Studies, 6(1), 21. https://doi.org/10.1186/ s40878-018-0088-0

Soskolne, V. (2001). Single parenthood, occupational drift and psychological distress among immigrant women from the former Soviet Union in Israel. Women \& Health, 33(3-4), 67-84.

Sossou, M.-A., Craig, C. D., Ogren, H., \& Schnak, M. (2008). A qualitative study of resilience factors of Bosnian refugee women resettled in the southern United States. Journal of Ethnic \& Cultural Diversity in Social Work, 17(4), 365-385.

Sundquist, J., Bayard-Burfield, L., Johansson, L. M., \& Johansson, S. E. (2000). Impact of ethnicity, violence and acculturation on displaced migrants: Psychological distress and 
psychosomatic complaints among refugees in Sweden. Journal of Nervous \& Mental Disease, 188(6), 357-365. https://doi. org/10.1097/00005053-200006000-00006

Turnip, S. S., Klungsøyr, O., \& Hauff, E. (2010). The mental health of populations directly and indirectly exposed to violent conflict in Indonesia. Conflict and Health, 4(1), 14.

Turnip, S. S., Sorbom, D., \& Hauff, E. (2016). Predicting positive mental health in internally displaced persons in Indonesia: The roles of economic improvement and exposure to violent conflict. Psychology, Health \& Medicine, 21(3), 286-294. https://doi.org/10. 1080/13548506.2015.1051554

Veronese, G., Pepe, A., Sala, G., Yamien, I., \& Vigliaroni, M. (2019). Positive experience, psychological functioning, and hope for the future as factors associated with mental health among young SubSaharan internally displaced people (IDP): A quantitative pilot study. International Journal of Mental Health, 1-23.

Wilson, A., \& Mittelmark, M. B. (2013). Resources for adjusting well to work migration: Women from northern Ghana working in Head Porterage in Greater Accra. Africa Today, 59(4), 25-38.

Ying, Y.-W., Lee, P. A., \& Tsai, J. L. (2000). Cultural orientation and racial discrimination: Predictors of coherence in Chinese American young adults. Journal of Community Psychology, 28(4), 427-441.

Ying, Y.-W., Lee, P. A., \& Tsai, J. L. (2007). Attachment, sense of coherence, and mental health among Chinese American college students: Variation by migration status. International Journal of Intercultural Relations, 31(5), 531-544.

Open Access This chapter is licensed under the terms of the Creative Commons Attribution 4.0 International License (http://creativecommons. org/licenses/by/4.0/), which permits use, sharing, adaptation, distribution and reproduction in any medium or format, as long as you give appropriate credit to the original author(s) and the source, provide a link to the Creative Commons license and indicate if changes were made.

The images or other third party material in this chapter are included in the chapter's Creative Commons license, unless indicated otherwise in a credit line to the material. If material is not included in the chapter's Creative Commons license and your intended use is not permitted by statutory regulation or exceeds the permitted use, you will need to obtain permission directly from the copyright holder. 